DE

M E D I C I N A

T R O P I C A L

$\mathrm{DE}$

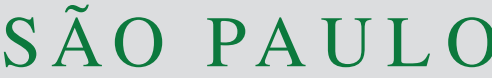

JOURNAL OF THE SÃO PAULO INSTITUTE OF TROPICAL MEDICINE

${ }^{1}$ Instituto Adolfo Lutz, Centro de Virologia, São Paulo, São Paulo, Brazil

2Universidade de São Paulo, Instituto de Ciências Biomédicas II, São Paulo, São Paulo, Brazil

Correspondence to: Maria Isabel de Oliveira

Instituto Adolfo Lutz, Centro de Virologia, Núcleo de Doenças Respiratórias, Av. Dr. Arnaldo 355, CEP 01246-902, São Paulo, SP, Brazil

Tel: +55 1130682906

E-mail: olive40@uol.com.br, maria.isabel@ial.sp.gov.br

Received: 13 May 2020

Accepted: 14 July 2020

\section{Frequency of congenital cytomegalovirus infections in newborns in the Sao Paulo State, 2010-2018}

\author{
Carla Grasso Figueiredo', Adriana Luchs', Edison Luiz Durigon², Danielle \\ Bruna Leal de Oliveira ${ }^{2}$, Vanessa Barbosa da Silva², Ralyria Melyria Mello², \\ Ana Maria Sardinha Afonso', Maria Isabel de Oliveira ${ }^{(1}$
}

\section{ABSTRACT}

Human cytomegalovirus (HCMV) infections remain a neglected public health issue. The aim of the present study was to evaluate the frequency of HCMV congenital infections in newborns up to 1 month in the Sao Paulo State, from 2010 to 2018. The molecular characterization of HCMV-positive samples was also undertaken. Urine samples from 275 potential congenital HCMV-infected patients were tested by real-time Polymerase Chain Reaction (qPCR). HCMV-positive samples were amplified by conventional PCR targeting the UL89 gene, sequenced and searched for mutations. A total of $32(11.6 \%)$ positiveHCMV cases were detected (mean Ct 30.59); mean and median age of 10.3 and 6 days old, respectively. Children aged between 0-3 weeks had higher HCMV detection rates $(84.4 \%$; 27/32). UL89 gene was successfully sequenced in two samples, both classified as the human betaherpesvirus 5 . No described resistance-associated mutations were identified. A routine screening in newborns coupled with the genetic characterization of key viral genes is vital to decrease sequels associated with congenital HCMV infections.

KEYWORDS: Congenital cytomegalovirus infection. Real-time PCR. Surveillance. DNA sequencing.

\section{INTRODUCTION}

Human Cytomegalovirus (HCMV) is a ubiquitous human-specific DNA virus, belonging to the Herpesviridae family ${ }^{1}$. A key characteristic of HCMV is its latency capacity, and after the primary infection HCMV can exit latency and re-enter the lytic lifecycle any time during the host life ${ }^{2}$. HCMV infection can be acquired through contamination with different biological fluids, and intrauterine transmission can occur in pregnant women without pre-existing immunity who acquire the primary infection HCMV infection in pregnancy or in women with pre-existing antibodies to HCMV through the reactivation of the primary infection or by acquiring a different HCMV strain ${ }^{3}$.

HCMV seroprevalence in adults ranges between $60 \%$ in developed countries and $100 \%$ in developing countries ${ }^{4}$. HCMV seropositivity in the Brazilian population range from $70 \%$ to $97 \%^{5-8}$. Congenital HCMV infection is the most common congenital infection worldwide, affecting $0.2 \%$ to $6.1 \%$ of live newborns ${ }^{1}$. The prevalence of congenital HCMV infection in Brazil is 1-8\%,

The diagnosis of the congenital HCMV infection should be conducted before the $3^{\text {rd }}$ week of life. After this, it is difficult to distinguish congenital infection from infection acquired in the postnatal period ${ }^{11}$. Traditionally, the virus isolation from 
urine or saliva samples in cell cultures has been the standard method for diagnosing the congenital HCMV infection. Recently, molecular techniques, as the polymerase chain reaction (PCR) and the real-time polymerase chain reaction (qPCR) has led to important advances in the congenital HCMV diagnosis ${ }^{12}$. The early diagnosis of congenital HCMV infection and the clinical follow-up are essential to detect and manage the disease and prevent sequels ${ }^{13}$. Despite its importance and the availability of rapid and specific molecular tests, numerous newborns suffering from congenital HCMV infection remain undiagnosed in Brazil as the public health system do not perform the screening of HCMV in regular bases ${ }^{10}$.

The purpose of the present study was to evaluate the frequency of HCMV congenital infections in newborns up to 1 month of age in the Sao Paulo State from 2010 to 2018. The qPCR technique was used to screen urine samples aiming to apply this approach in future screening programs for HCMV congenital infections in the Brazilian Health System (SUS). In addition, molecular characterization of HCMV positive samples was also undertaken.

\section{METHODS}

This is a retrospective and descriptive study conducted with urine samples collected from newborns $(<1$ month old). Urine samples were obtained from potential congenital HCMV-infected patients at Brazilian Unified Health System (SUS) units following medical request, and sent to the Respiratory Diseases Laboratory of Adolfo Lutz Institute, a reference center for HCMV surveillance in the Sao Paulo State. The present investigation was funded between collaborating institutes involved in public or nonpublic HCMV surveillance programs: (i) Regional Health Departments (DRS I to XVII), (ii) Clinical Hospital of Sao Paulo, (iii) Emilio Ribas Hospital, (iv) University Hospital of Sao Paulo and (v) Santa Casa Hospital of Sao Paulo. The routine of diagnostic tests conducted for HCMV at the Respiratory Diseases Laboratory includes more complex methodologies, such as viral isolation in cell cultures, conventional polymerase chain reaction (PCR), real-time PCR (qPCR), and eventually, DNA sequencing. Serological tests to detect IgM and/or IgG are conducted at the requesting units. From 2010 to 2018, a total of 1,279 urine samples were received at the Respiratory Diseases Laboratory and the original urine samples were preserved at $-70{ }^{\circ} \mathrm{C}$. A total of 275 samples were randomly selected based on IgM-positive results and radiological exams suggestive of HCMV infection according to data retrieved from the medical records; and preserved specimen's availability. All the 275 urine samples were tested for the presence of
HCMV exclusively by qPCR methodology as no other biological specimens belonging to the same selected patient were available for testing.

Viral DNA was extracted using the QIAamp ${ }^{\circledR}$ DNA Mini Kit (QIAGEN Valencia, CA, USA), according the manufacturer's protocol. The qPCR for the majorimmediate early (MIE) gene was performed according to a previously described protocol ${ }^{14}$, generating amplicons of 76 bp. As a control to rule out the presence of PCR inhibitors, and to monitor the nucleic acid extraction efficiency, each sample was tested by qRT-PCR for the presence of the human ribonuclease (RNase) P gene following the protocol described by Emery et al. ${ }^{15}$. The threshold cycle $(\mathrm{Ct})$ value from this qPCR was used as a proxy measure of the HCMV load. The $\mathrm{Ct}$ value is inversely proportional to the amount of virus present in the sample, so the lower the $\mathrm{Ct}$ value the higher the viral load ${ }^{16}$. Samples were considered positive when a $\mathrm{Ct}$ value $\leq 39$ was obtained ${ }^{14}$. HCMV-positive samples were subsequently targeted for amplification by a conventional PCR with HCMV-specific primers 707s and 707as, amplifying a 519-nucleotide (nt) portion of the DNA packaging terminase subunit 1 (UL89), as described elsewhere ${ }^{17,18}$.

PCR amplicons were subjected to purification using the ExoSAP-IT $^{\mathrm{TM}}$ Kit (Applied Biosystems, Foster City, CA, USA), and sequenced using the BigDye ${ }^{\mathrm{TM}} \mathrm{Kit}$ v3.1 (Applied Biosystems, Foster City, CA, USA) with the same primer pairs used for $\mathrm{PCR}^{18}$. Dye-labeled products were sequenced using an ABI 3130 sequencer (Applied Biosystems, Inc., Foster city, CA, USA), following purification using Centri$\mathrm{Sep}^{\mathrm{TM}}$ spin columns (Invitrogen, Carlsbad, CA, USA). Sequencing chromatograms were edited manually using the Sequencher 4.7 software (Gene Codes Corporation, Ann Arbor, MI, USA). Sequences were screened on the NCBI website using the Basic Local Alignment Search Tool (BLAST) to assign the types of the studied strains. The typing verification was conducted using the MEGA software version 6.0 (NCBI, Bethesda, MD, USA). Prototype HCMV sequences, available in GenBank were aligned together with the sequences generated here using the BioEdit software, version 7.0.5.2 (Ibis Therapeutics, USA). Neighbor Joining (NJ) trees were constructed based on a Kimura 2-parameter model determined by MEGA 6.0 with 1000 bootstrap replicates ${ }^{19}$. Nucleotide sequences determined in this study were deposited in GenBank under the accession $\mathrm{N}^{\circ}$ MT345567 and MT447555. The publically available web-based search tool MRA-mutation resistance analyzer ${ }^{20}$ was used to identify possible mutations associated with drug resistance.

The ethical approval was granted by Adolfo Lutz Institute (CEP-IAL 2.984.740, CAAE 96782618.5.0000.0059). This 
was an anonymous unlinked study and informed consent was not required according to the Resolution $\mathrm{N}^{\circ} 466 / 12$ concerning research involving human beings (Conselho Nacional de Saude (CNS)/Ministerio da Saude (MS), Brasilia, 2012).

\section{RESULTS AND DISCUSSION}

Of the 275 urine samples tested, 32 specimens (11.6\%) were positive for HCMV by qPCR targeting the MIE gene (mean Ct value of 30.59). The mean age of the newborns was 10.3 days (range: 2 days to 30 days), while the median age was 6 days old. Newborns aged between 0 and 3 weeks had higher detection rates of HCMV $(84.4 \%$; $27 / 32$; mean $\mathrm{Ct}=30.62)$ than infants in the age group older than 3 weeks ( $15.6 \%$; $5 / 32$; mean $\mathrm{Ct}=30.40)$, emphasizing the importance of the early specimen collection (under 3 weeks of life) for the accurate diagnosis ${ }^{21}$. After this period, the assessment of the viral infection origin is impaired as the contagion could have occurred throughout the birth canal, saliva or breast milk ${ }^{10}$. No significant differences were observed between HCMV Ct levels and the newborns age. HCMV infections were detected throughout the study period (from 2010 to 2018), suggesting that urine samples frozen at $-70{ }^{\circ} \mathrm{C}$ were stable for a long-term storage (around 10 years) after collection. It is well known that viruses can be stored and preserved at low or ultra-low temperatures as an infectious material for long periods of time ${ }^{22}$.

The frequency of congenital HCMV infections detected here $(11.6 \%)$ was similar to the one observed in other study carried out in Japan (10\%) using conventional PCR in urine samples ${ }^{23}$. However, the infection rate was higher in comparison with Portugal (0.4\%) and Belgium (0.5\%) using viral isolation in cell cultures from urine samples ${ }^{24,25}$; the USA $(0.5 \%)$ using qPCR in saliva specimens targeting the glycoprotein $\mathrm{B}(\mathrm{gB})$ and the immediate early gene 2 (IE-2) exon 5 region $^{12}$; and with the sub-Saharan Africa (3.8\%) using qPCR in saliva, serum and urine samples targeting the phosphoprotein pp65 (UL83) ${ }^{26}$. Lower prevalences have also been reported in previous studies conducted in Brazil: Sao Paulo (1\%) (1980-1982) using viral isolation in cell culture from urine samples and specific immunofluorescence anti-HCMV IgM in umbilical-cord serum $^{27}$, Belo Horizonte (6.8\%) (1995-1998) and Porto Alegre $(0.8 \%)$ (2003) using conventional PCR in urine samples ${ }^{28,29}$; Ribeirao Preto (1\%) (2003-2009) and Ilheus (1.2\%) (2010-2012) using conventional PCR in urine and/ or saliva samples ${ }^{9,10}$. This observed disagreement may be mostly associated with differences in the diagnostic methods and clinical samples ${ }^{10,24}$. It is important to note that in-house qPCR is not a standardized methodology and different primers, target genes and amplification cycles can be used for HCMV detection ${ }^{12,14,26}$, making comparison between studies rather complex. Divergence in prevalence rates might also be linked to the study design, recruitment criteria, settings, the number of specimens tested, and the socioeconomic status of the population ${ }^{10,24}$. An investigation conducted by Pannuti et al. ${ }^{27}$ suggested that in populations with unfavorable socioeconomically conditions, congenital HCMV infection tends to be more prevalent. This 9-year retrospective study helped to clarify the HCMV burden in congenital infections in the Sao Paulo State.

Regarding the 32 HCMV-positive samples, the UL89 gene could be amplified in six samples $(18.7 \% ; 6 / 32)$, but only two were successfully sequenced (2/6 or $33.3 \%)$. It is well known that qPCR provide a rapid diagnosis with increased sensitivity for the HCMV detection ${ }^{14}$. However, the molecular characterization depends mainly on conventional PCR followed by Sanger sequencing, which clearly impaired our capacity in identifying all the HCMV-positive samples.

The typing verification and the phylogenetic analysis was performed with the $510 \mathrm{bp}$ DNA packaging terminase subunit 1 (UL89) fragment. The genetic trees include the strains sequenced in this study (highlighted in bold) along with prototype sequences obtained from GenBank. Both samples (IAL-230 and IAL-259) were classified as Human betaherpesvirus 5 (HHV-5), forming a wellsupported monophyletic group (Figure 1). The comparison of nucleotide sequences between the two Brazilian isolates showed a maximum identity of $100 \%$ (100\% aa), with similar high values also observed following the comparison with other HHV-5 strains (94.3\% nt, 93.9-100\% aa) identified worldwide.

Recent attempts to develop new anti-HCMV compounds have focused on the viral terminase complex involved in the viral DNA cleavage/packaging. The terminase complex is highly HCMV-specific, with no counterpart in mammalian cells, thus representing a target of choice for new antivirals ${ }^{30}$. The small subunit of the HCMV terminase complex is encoded by the Open Reading Frame - ORF UL89 ${ }^{31}$, and multiple mutations have been detected in this ORF of drug-resistant isolates ${ }^{32}$. No described resistanceassociated mutations within the UL89 gene were identified by the MRA-mutation resistance analyzer web tool in the Brazilian HCMV samples. Nevertheless, several mutations of yet unknown clinical significance were found (Figure 2). A large number of new mutations of undetermined medical implication has been identified in either clinical or laboratory isolates ${ }^{33}$; however their impact on the HCMV treatment remain to be evaluated.

Despite the recognized importance of the congenital 


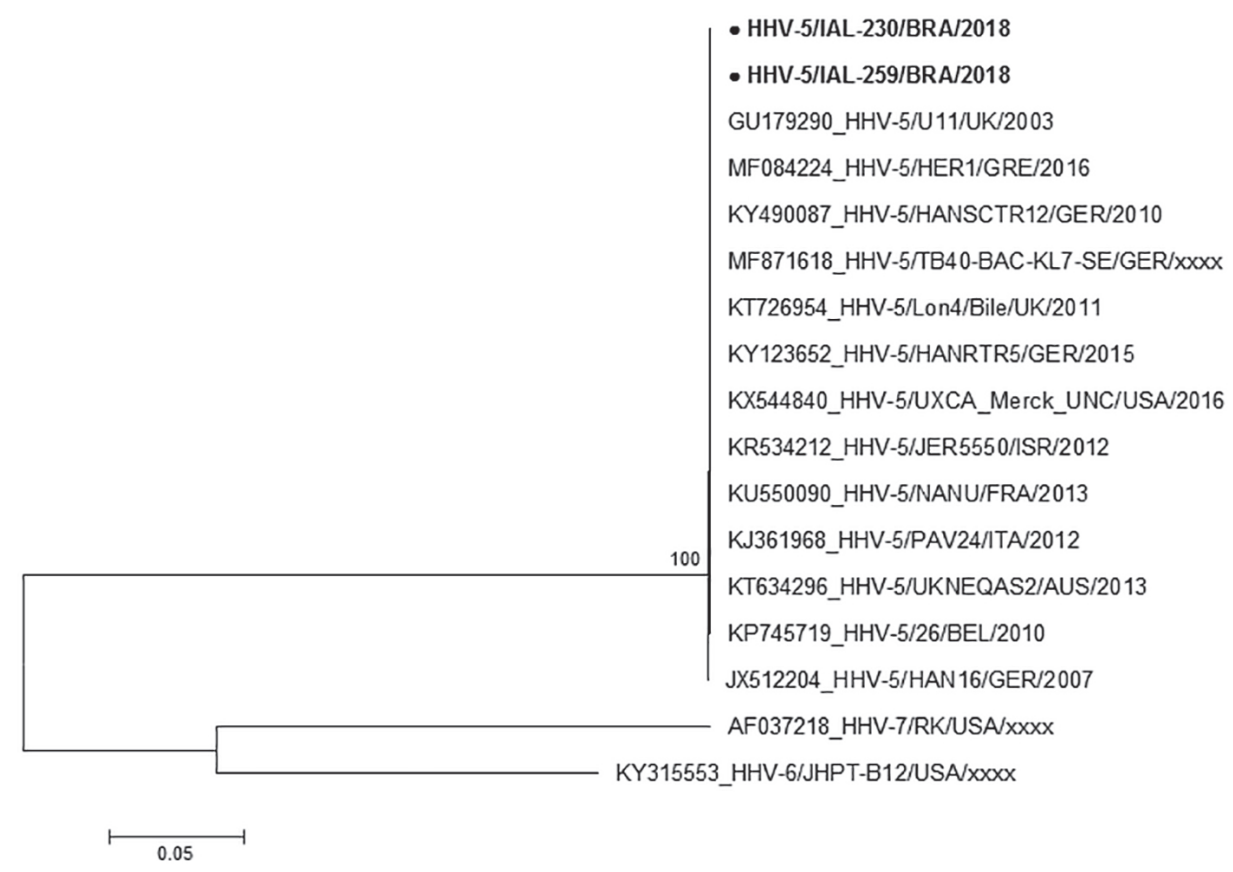

Figure 1 - Nucleotide sequence-based similarity analysis of the Brazilian Human betaherpesvirus 5 (HHV-5) DNA packaging terminase subunit 1 (UL89) (indicated in bold) with other selected HCMV strains. A neighbor-joining tree of partial UL89 ORF sequences was generated using the MEGA 6.0 software. Reference HHV-5 to -7 strains were obtained from the GenBank database. The accession number, isolates, country and year of each strain are indicated. The scale indicates the number of divergent nucleotide residues. Bootstrap values are shown as percentages at the branch nodes.

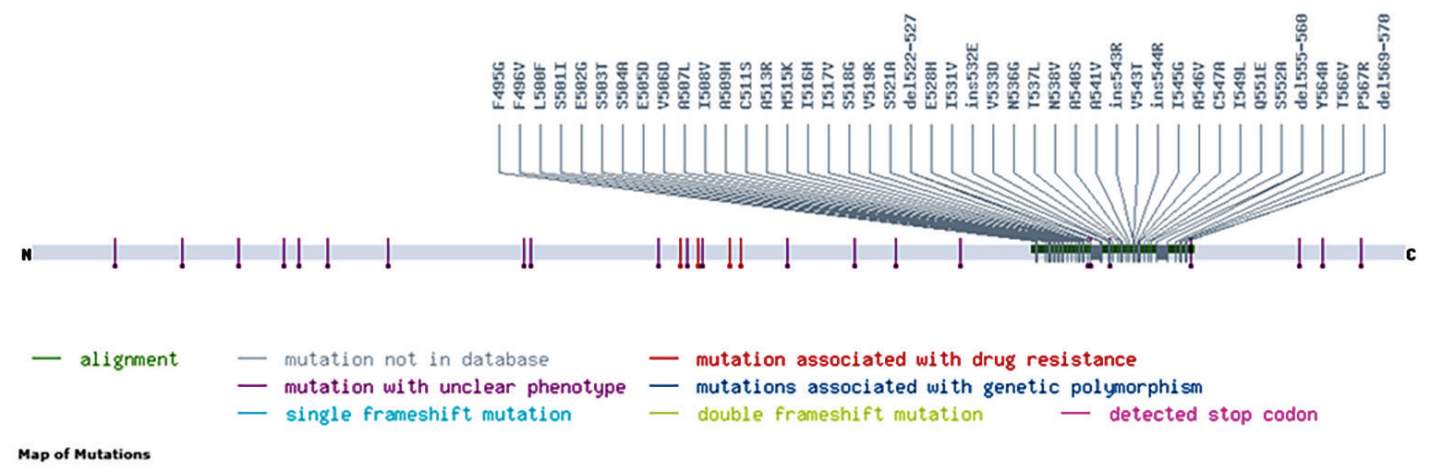

Figure 2 - Map of mutations (substitutions, insertions and deletions) identified in DNA packaging terminase subunit 1 (UL89) partial ORF gene sequences of the Brazilian Human betaherpesvirus 5 (HHV-5) IAL-230 and IAL-259 strains using the MRA-mutation resistance analyzer web tool ${ }^{20}$.

HCMV infection, it is still a wide neglected syndrome in Brazil. Significant advances in diagnostic techniques have occurred in the last years. Real time PCR is highly sensitive and could be applied in large scale. The implementation of a routine screening policy in newborns coupled with the genetic characterization of key viral genes is an important strategy focusing on decreasing the sequels associated with congenital HCMV infections.

\section{AUTHORS' CONTRIBUTIONS}

MIO conceived the study; ELD, DBLO, AMSA and MIO designed the study protocol.; CGF, AL, VBS, RMM and MIO participated in the conduct of the study; CGF and $\mathrm{MIO}$ acquired the data and performed the PCR assays; CGF, AL and MIO conducted the sequencing assays; CGF, AL, ELD, DBLO, VBS, RMM and MIO analyzed and interpreted the data; $\mathrm{AL}$ and MIO drafted the manuscript; CGF, ELD, DBLO, AMSA, VBS and RMM critically revised the manuscript for intellectual content. All authors read and approved the final version. MIO is guarantors of the paper.

\section{CONFLICT OF INTERESTS}

The authors have no conflict of interests to disclose. 


\section{FUNDING}

This study was partially supported by Graduate Program in Science, Coordinator for Disease Control; Ministry of Health-PPG-CCD-SES/SP. Carla Grasso Figueiredo was supported by a fellowship of the Association for the Improvement of Higher Education Personnel (CAPES).

\section{REFERENCES}

1. Marsico C, Kimberlin DW. Congenital Cytomegalovirus infection: advances and challenges in diagnosis, prevention and treatment. Ital J Pediatr. 2017;43:38.

2. Dupont L, Reeves MB. Cytomegalovirus latency and reactivation: recent insights into an age old problem. Rev Med Virol. 2016;26:75-89.

3. Davis NL, King CC, Kourtis AP. Cytomegalovirus infection in pregnancy. Birth Defects Res. 2017;109:336-46.

4. Griffiths P, Baraniak I, Reeves M. The pathogenesis of human cytomegalovirus. J Pathol. 2015;235:288-97.

5. Suassuna JH, Leite LL, Villela LH. Prevalence of cytomegalovirus infection in different patient groups of an urban university in Brazil. Rev Soc Bras Med Trop. 1995;28:105-8.

6. Amaral RP, Amaral RP, Saidneuy AE, Ribeiro WL, Andrade J. Serological profile of potential solid organ donors in Santa Catarina, Brazil. Transplant Proc. 2008;40:665-7.

7. Guerra AB, Siravenha LQ, Laurentino RV, Feitosa RN, Azevedo VN, Vallinoto AC, et al. Seroprevalence of HIV, HTLV, CMV, $\mathrm{HBV}$ and rubella virus infections in pregnant adolescents who received care in the city of Belém, Pará, Northern Brazil. BMC Pregnancy Childbirth. 2018;18:169.

8. Tiguman GM, Poll LB, Alves CE, Pontes GS, Silva MT, Galvao TF. Seroprevalence of cytomegalovirus and its coinfection with Epstein-Barr virus in adult residents from Manaus: a population-based study. Rev Soc Bras Med Trop. 2020;53:e20190363.

9. Mussi-Pinhata MM, Yamamoto AY, Moura Brito RM, Lima Isaac M, Carvalho e Oliveira PF, Boppana S, et al. Birth prevalence and natural history of congenital cytomegalovirus infection in a highly seroimmune population. Clin Infect Dis. 2009;49:522-8.

10. Marin LJ, Santos de Carvalho Cardoso E, Bispo Sousa SM, Debortoli de Carvalho L, Marques Filho MF, Raiol MR, et al. Prevalence and clinical aspects of CMV congenital infection in a low-income population. Virol J. 2016;13:148.

11. Fowler KB, Ross SA. Congenital viral infections continue to affect neonates. Lancet Infect Dis. 2020;20:152-3.

12. Boppana SB, Ross SA, Shimamura M, Palmer AL, Ahmed A, Michaels MG, et al. Saliva polymerase-chain-reaction assay for cytomegalovirus screening in newborns. N Engl J Med. 2011;364:2111-8

13. Yamamoto AY, Mussi-Pinhata MM, Marin LJ, Brito RM, Oliveira
PF, Coelho TB. Is saliva as reliable as urine for detection of cytomegalovirus DNA for neonatal screening of congenital CMV infection? J Clin Virol. 2006;36:228-30.

14. Watzinger F, Suda M, Preuner S, Baumgartinger R, Ebner K, Baskova L, et al. Real-time quantitative PCR assays for detection and monitoring of pathogenic human viruses in immunosuppressed pediatric patients. J Clin Microbiol. 2004;42:5189-98.

15. Emery SL, Erdman DD, Bowen MD, Newton BR, Winchell JM, Meyer RF, et al. Real-time reverse transcription-polymerase chain reaction assay for SARS-associated coronavirus. Emerg Infect Dis. 2004;10:311-6.

16. Phillips G, Lopman B, Tam CC, Iturriza-Gomara M, Brown D, Gray J. Diagnosing norovirus-associated infectious intestinal disease using viral load. BMC Infect Dis. 2009;9:63.

17. Chmielewicz B, Goltz M, Ehlers B. Detection and multigenic characterization of a novel gammaherpesvirus in goats. Virus Res. 2001;75:87-94.

18. Hargis AM, Ginn PE, Mansell JE, Garber RL. Ulcerative facial and nasal dermatitis and stomatitis in cats associated with feline herpesvirus 1. Vet Dermatol. 1999;10:267-74.

19. Tamura K, Stecher G, Peterson D, Filipski A, Kumar S. MEGA6: molecular evolutionary genetics analysis version 6.0. Mol Biol Evol. 2013;30:2725-9.

20. Kestler HA. HCMV drug resistance mutations. [cited $2020 \mathrm{Jul}$ 16]. Available from: https://www.informatik.uni-ulm.de/ni/ staff/HKestler/hcmv/

21. Ebrahimi-Rad M, Shakeri TS, Shirvani F, Shahrokhi K, Shahrokhi $\mathrm{N}$. Prevalence of congenital cytomegalovirus infection in symptomatic newborns under 3 weeks in Tehran, Iran. BMC Infect Dis. 2017;17:688.

22. Gould EA. Methods for long-term virus preservation. Mol Biotechnol. 1999;13:57-66.

23. Oda K, Oki S, Tsumura N, Nakao M, Motohiro T, Kato H. Detection of cytomegalovirus DNA in urine from newborns in NICU using a polymerase chain reaction. Kurume Med J. 1995;42:39-44.

24. Almeida S, Gouveia P, Jorge A, Mendes A, Duarte C, Faria N, et al. Infecção congénita por Citomegalovirus. Prevalência numa população da Beira Interior. Acta Pediatr Port. 2010;41:162-5.

25. Foulon I, Naessens A, Foulon W, Casteels A, Gordts F. A 10 -year prospective study of sensorineural hearing loss in children with congenital cytomegalovirus infection. J Pediatr. 2008; $153: 84-8$.

26. Mwaanza N, Chilukutu L, Tembo J, Kabwe M, Musonda K, Kapasa M, et al. High rates of congenital cytomegalovirus infection linked with maternal HIV infection among neonatal admissions at a large referral center in sub-Saharan Africa. Clin Infect Dis. 2014;58:728-35.

27. Pannuti CS, Vilas-Boas LS, Angelo MJ, Carvalho RP, Segre CM. Congenital cytomegalovirus infection. Occurrence in two 
socioeconomically distinct populations of developing country. Rev Inst Med Trop Sao Paulo. 1985;27:105-7.

28. Santos DV, Souza MM, Gonçalves SH, Cotta AC, Melo LA, Andrade GM, et al. Congenital cytomegalovirus infection in a neonatal intensive care unit in Brazil evaluated by PCR and association with perinatal aspects. Rev Inst Med Trop Sao Paulo. 2000;42:129-32.

29. Miura CS, Miura E, Mombach AB, Chesky M. The prevalence of congenital cytomegalovirus infection in newborn infants at an intensive care unit in a public hospital. J Pediatr (Rio J). 2006;82:46-50.

30. Ligat G, Cazal R, Hantz S, Alain S. The human cytomegalovirus terminase complex as an antiviral target: a close-up view. FEMS Microbiol Rev. 2018;42:137-45.
31. Thoma C, Borst E, Messerle M, Rieger M, Hwang JS, Bogner E. Identification of the interaction domain of the small terminase subunit pUL89 with the large subunit pUL56 of human cytomegalovirus. Biochemistry. 2006;45:8855-63.

32. Buerger I, Reefschlaeger J, Bender W, Eckenberg P, Popp A, Weber $\mathrm{O}$, et al. A novel nonnucleoside inhibitor specifically targets cytomegalovirus DNA maturation via the UL89 and UL56 gene products. J Virol. 2001;75:9077-86.

33. Komatsu TE, Pikis A, Naeger LK, Harrington PR. Resistance of human cytomegalovirus to ganciclovir/valganciclovir: a comprehensive review of putative resistance pathways. Antiviral Res. 2014;101:12-25. 